\title{
NUMERICAL PREDICTION OF EROSIVE COLLAPSE EVENTS IN UNSTEADY COMPRESSIBLE CAVITATING FLOWS \\ MARINE 2011
}

\author{
MICHAEL S. MIHATSCH ${ }^{*}$, STEFFEN J. SCHMIDT ${ }^{*}$, MATTHIAS THALHAMER \\ AND NIKOLAUS A. ADAMS* \\ * Institute of Aerodynamics and Fluid Mechanics \\ Technische Universität München \\ Boltzmannstr. 15, D-85748 Garching, Germany \\ e-mail: michael.mihatsch@aer.mw.tum.de,web page: http://www.aer.mw.tum.de/en/home/
}

Key words: Cavitation, Erosion, Numerical Simulation, Multiphase Flow

Summary. The objective of the present investigation is the numerical prediction of the potential of a flow to inflict surface damage by cavitation. For this purpose, physical criteria are derived that detect and quantify relevant flow phenomena. In particular, we present a numerical approach for tracing isolated collapses of vapor clouds during the numerical simulation of the flow. The suggested "collapse detector" provides the frequency of collapses, their positions and resulting maximum pressures as well as the maximum condensation rate of each event. These data, together with the maximum wall pressure, allow for an automatic indication of erosion-sensitive areas.

The employed flow solver CATUM (CAvitation Technische Universität München) is a density-based 3-D finite volume method equipped with a Low-Mach-number consistent flux function. All fluid components (liquid, vapor, saturated mixture) are modeled by closed form equations of state.

To assess the novel approach we simulate an experimentally investigated nozzle-target flow. A comparison of numerically predicted collapse events with the experimentally observed areas of cavitation erosion substantiates the proposed methodology. The obtained data represent a time-history of collapse events together with their position and strength and may be used to estimate erosion rates.

\section{INTRODUCTION}

Hydrodynamic cavitation and cavitation erosion are well-known phenomena in naval engineering ${ }^{1,2}$. Distinctive examples include cavitation on suction sides of propeller blades and cavitating tip vortices. The basic mechanisms of hydrodynamic cavitation are flow- 
induced evaporation due to the pressure drop of an accelerated liquid, and inertia controlled recondensation of liquid-embedded vapor bubbles due to pressure recovery. The collapse-like recondensation results in the generation of shock waves that are supposed to constitute the main mechanism of cavitation erosion. These shocks propagate approximately with the speed of sound $c_{l}=\mathrm{O}\left(10^{3}\right) \mathrm{m} / \mathrm{s}$ of the liquid and their intrinsic time scale is about 2 orders of magnitude smaller than the time scale of the underlying shear flow.

Since the simulation of erosive collapses in cavitating flows requires the resolution of both time scales, time step sizes of the order of nanoseconds are inevitable and millions of time steps have to be computed to cover the characteristic time interval of the shear flow. It is neither reasonable to store the enormous amount of data generated by such a simulation, nor is it feasible to evaluate manually the data with respect to collapse characteristics and erosion aggressiveness. Instead, new approaches have to be developed that allow for automatic detection and evaluation of aggressive collapse events predicted by 3-D unsteady simulations of cavitating flows.

\section{UNDERLYING NUMERICAL MODEL}

We apply our flow simulation code CATUM (CAvitation Technische Universität München ${ }^{3}$ ), which is a density based finite volume method employing a Low-Mach-number consistent flux function and an explicit time marching procedure. The spatial reconstruction of the velocity field is a WENO-3 procedure; density and internal energy are reconstructed by monotonic TVD limiters ("minmod"). Time marching is performed through an explicit low storage 4-step Runge-Kutta method with an optimized stability region. The combination of both methods results in a $2^{\text {nd }}$ order numerical approach in space and time for smooth flow, and it ensures a sharp representation of discontinuous flow features such as shocks and contact waves. In this investigation we focus on the simulation of inertia-driven effects and wave dynamics and we neglect viscous effects. Hence, the governing equations are the compressible Euler equations.

In the present investigation the thermodynamic properties of the working fluid, in this case water, are characterized by closed-form equations of state: the liquid phase is specified by a modified Tait law, saturated two-phase regions are modeled by polynomial fits of the IAPWS $^{4}$ data and pure vapor is modeled by an modified ideal gas law. In order to allow for the simulation of shock formation and wave propagation, the compressibility of the fluids (liquid and vapor) is taken into account. Therefore, the numerical time step is necessarily proportional to the ratio of the smallest length scale (minimum grid size) and the fastest signal speed ( $\sim$ speed of sound of the liquid).

The phase transition model is based on local equilibrium assumptions for pressure, temperature, and specific Gibbs functions. We have observed that this assumption is justified since most of technically relevant hydrodynamic cavitation processes occur close to the equilibrium vapor pressure. Although this observation is questionable for incipient cavitation, it applies to developed cavitating flows, such as the one investigated within the presented research work. Previous investigations show that CATUM is able to predict even delicate flow features, such as irregular break-up patterns of partial cavities of developed cavitating flows. ${ }^{5}$ 
We adopt body-fitted hexahedral grids to discretize the flow field. The data structure is based on local blocks and a pre-processor load balance strategy is utilized to obtain approximately linear scaling on multi-processor systems.

\section{MODELING AND IMPLEMENTATION OF EROSION INDICATORS AND COLLAPSE DETECTORS}

Shock waves initiated by violent cloud collapses can generate intense pressure peaks on the order of several thousand bars. If such a shock wave is emitted sufficiently close to a solid surface, its impact on the solid material may result in dynamic loads that lead to material fatigue. Thus, instantaneous maximum loads may provide an indication of locations where erosion is likely to occur. Therefore monitoring the maximum pressure arising in each computational cell during the whole simulation is one efficient approach for detecting erosion sensitive areas within the flow field and especially along solid walls. Although this procedure was applied successfully in previous investigations ${ }^{5}$, it has several obvious drawbacks: The approach neither provides information about the frequency of collapse events, nor does it allow to distinguish between stagnation pressures and instantaneous maximum loads with durations of only a few microseconds. However, even this limited amount of achievable information allows for the indication of erosion-sensitive areas.

Our recent development focuses on a more detailed approach that we denote as "collapse detector". The concept involves the derivation of a set of physical criteria to detect collapses of isolated clouds and to characterize the strength of the generated shock waves as predicted by our numerical simulations. Figure 1 shows a schematic of consecutive stages of a cloudcollapse. In the lower part of this figure the average divergence of the velocity field around the collapsing cloud is depicted.

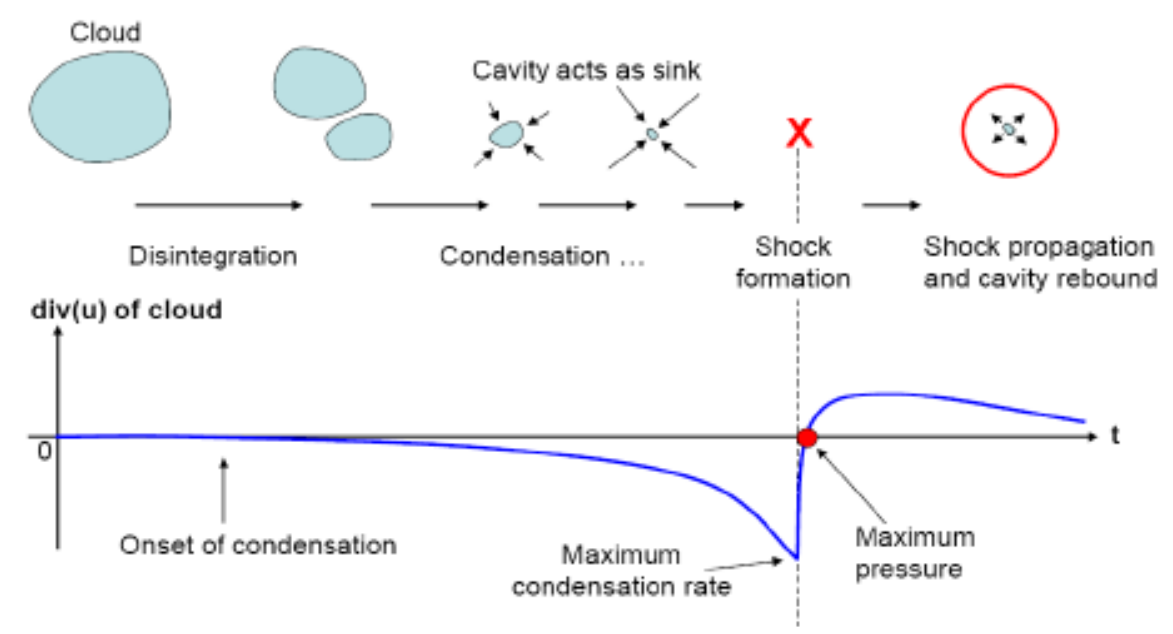

Figure 1: Sketch of consecutive stages of the collapse of a vapor cloud together with the temporal evolution of the divergence of the velocity field.

The onset of condensation results in the formation of a velocity field that is approximately directed towards the center of the cloud - similar to an elementary sink flow. Thus, the divergence of the velocity field is negative and decreasing. Since we assume that the specific 
Gibbs functions remain in equilibrium, and due to disregarding solved or dissolved gas, the pressure within the cloud remains approximately constant. Hence the surrounding liquid is further accelerated towards the center of the cloud until the vapor completely condenses at the last stage of the collapse. At that point the inertia of this sink flow results in a massive increase of the pressure. The maximum pressure is reached when the mass flow towards the center vanishes. At that point, the divergence changes its sign and the negative radial pressure gradient leads to an outward directed velocity field. Consequently, the pressure at the center decreases and may even lead to a re-evaporation of the liquid (rebound of the cavity). These considerations motivate the following definition of a numerical "collapse detector":

* We denote as "candidates" such computational cells where the vapor volume content condenses completely during the last time step. If the surrounding cells of a "candidate" contain liquid only, an isolated collapse is detected.

* Once a collapse is detected, the maximum pressure is generated at that instant in time when the divergence of the velocity field changes its sign.

* The strength of the collapse is characterized by its maximum (negative) divergence and its maximum pressure.

The main advantages of the "collapse detector" as compared to the maximum pressure approach are as follows:

* The collapse detector automatically distinguishes between collapse-induced maximum pressures and high pressures at stagnation points or due to wave interaction.

* The number of collapse events, as well as their position, and their strength provide important information about a possible stress profile the material is exposed to. This information can be used to estimate erosion rates.

An apparent deficiency of the "collapse detector" could be the missing information of the collapse intensity at the material surfaces. To overcome this drawback, an efficient projection method is proposed. We adopt the linear decay law of spherical waves to estimate the resulting pressure at the wall $p_{\text {wall }}$ from the known maximum pressure in the collapse center $p_{\text {collapse }}$ and from the distance of the collapse to the wall $r_{\text {wall }}$. The linear decay law states that the amplitude of a linear spherical pressure wave is inversely proportional to the radial distance of the wave front measured from its origin, hence $p(r) \sim 1 / r$. We assume that the initial radius of the wave front is proportional to the cube root of the volume $\mathrm{V}_{\text {cell }}$ of the cell where the collapse was detected. Thus, we obtain

$$
p_{\text {wall }} \sim \frac{\sqrt[3]{V_{\text {cell }}}}{r_{\text {wall }}} p_{\text {collapse }}
$$

This concept allows for the reduction of grid dependencies of the predicted maximum pressures as well. Instead of the maximum pressure, the value $p_{\text {collapse }} \cdot \sqrt[3]{V_{\text {cell }}}$ may be used to characterize the strength of a collapse. Preliminary investigations demonstrate the applicability of this approach., 


\section{RESULTS}

We simulate an experimentally investigated nozzle-target flow $^{8}$ to evaluate the numerical predictability of erosion-sensitive areas. The experimental setup results in an axisymmetric stagnation flow as shown in Figure 2. At the exit of the nozzle the fluid accelerates along a small radius and forms a radial cavitation pocket. Collapse-induced erosion is observed experimentally within an annulus at the surface of the target disc. The remaining parts are manufactured out of highly resistant material.

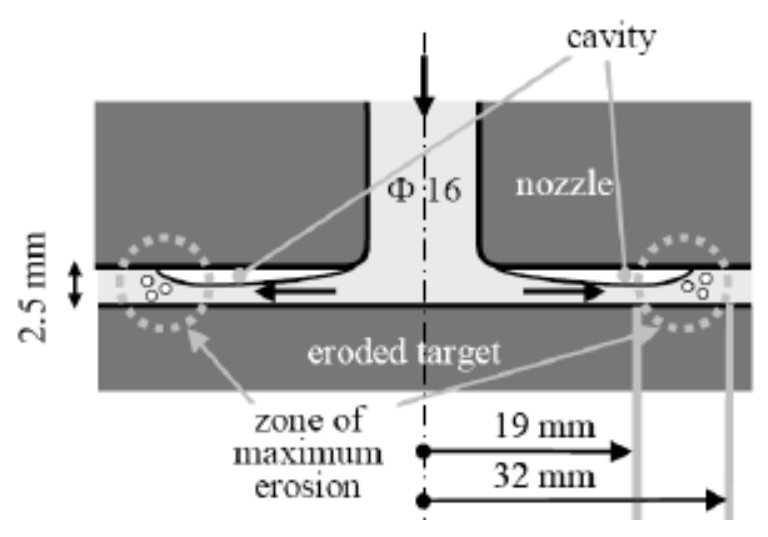

Figure 2: Sketch of the experimental setup. ${ }^{8}$

Figure 3 shows a cut through the computational domain. In accordance with the experiment, water at approximately room temperature enters the nozzle with the inlet-velocity $\mathrm{u}_{\mathrm{in}}=31 \mathrm{~m} / \mathrm{s}$. An asymptotic pressure boundary condition $\mathrm{p}_{\mathrm{e}}=10.1$ bar is imposed at the exit of a circular reservoir attached at a radial distance of $100 \mathrm{~mm}$. All solid boundaries are treated as inviscid adiabatic walls. The computational grid consists of $5 \cdot 10^{5}$ body-fitted hexahedrons structured in 56 blocks.

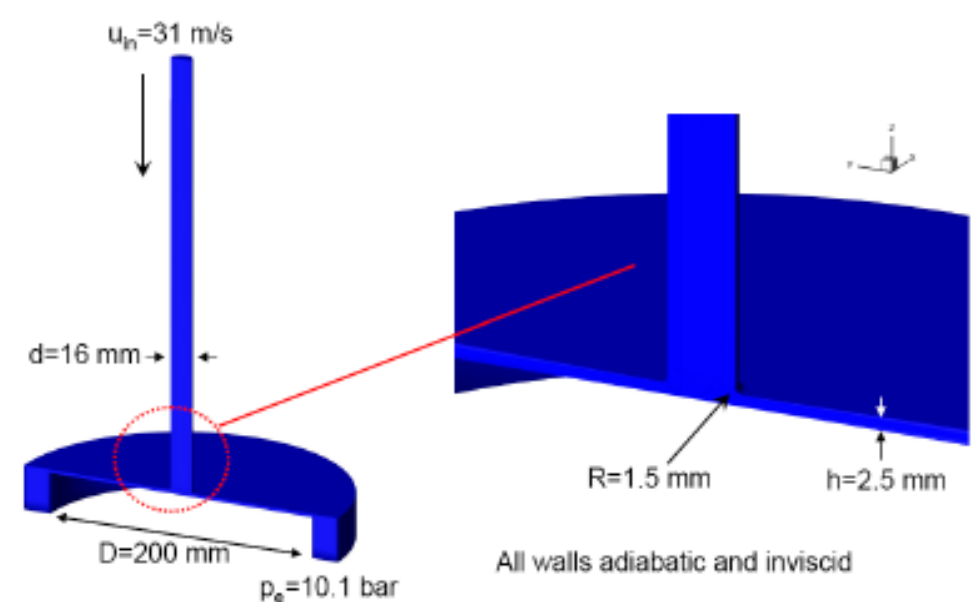

Figure 3: Cut through the numerical domain and imposed boundary conditions. The grid consists of $5 \cdot 10^{5}$ cells in 56 blocks and the numerical time step size is $\Delta \mathrm{t} \approx 2.8 \cdot 10^{-8} \mathrm{~s}$. The simulation is performed for the whole $360^{\circ}$ domain. 
Figure 4 shows the time history of the vapor volume fraction within the computational domain. The time intervals 1 and 2 correspond to a grid-sequencing-technique where the numerical solution is initially obtained on a coarse grid (interval 1) and interpolated to the fine grid. Only a short time interval (interval 2) is required until the solution on the fine grid establishes. The analyzed part of the simulation (interval 3 ) consists of $2.5 \cdot 10^{6}$ time steps with a time step-size of $\Delta \mathrm{t} \approx 2.8 \cdot 10^{-8} \mathrm{~s}$. This corresponds to a simulated physical time interval of $7 \cdot 10^{-2} \mathrm{~s}$. The computation required $3920 \mathrm{CPU}$-hours ( 70 hours on 56 cores, Intel Nehalem-EP based 8-way nodes).

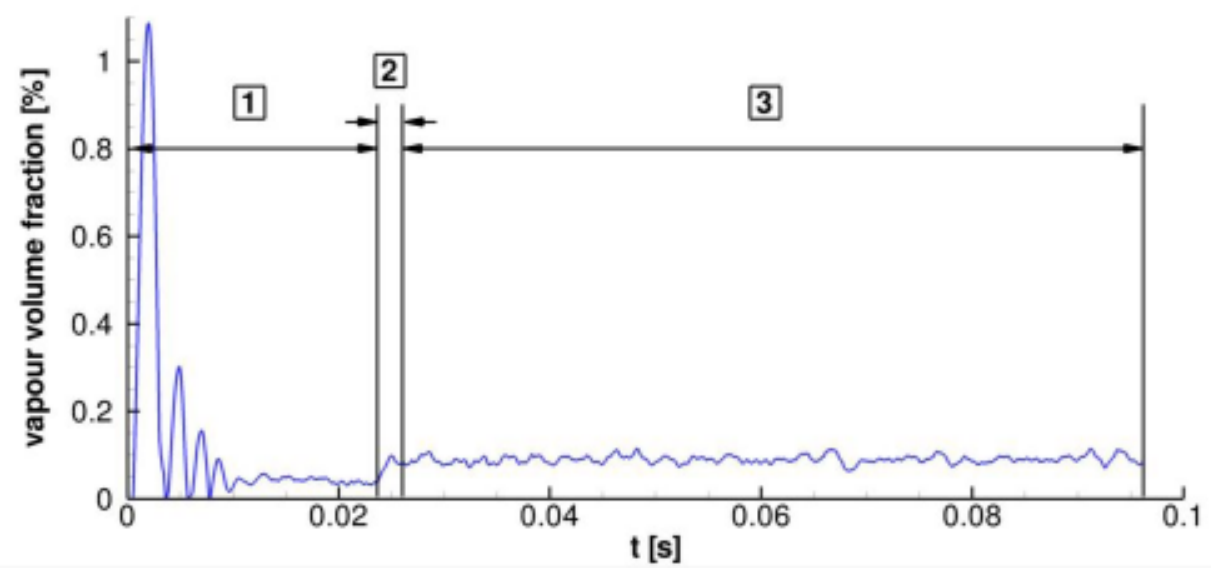

Figure 4: Time history of the vapor volume fraction referred to the volume of the whole computational domain. Interval 1: Preliminary calculation on a coarse grid. Interval 2: Interpolation to the fine grid. Interval 3: Analyzed part of the simulation on the fine grid.

Figure 6 shows instantaneous vapor structures at 4 equidistant instants in time $\left(\Delta \mathrm{t}=8.4 \cdot 10^{-5} \mathrm{~s}\right)$. Blue iso-surfaces correspond to a vapor volume fraction of $\alpha=0.1$. We observe the onset of sheet-cavitation at the rounding of the nozzle outlet. The fragmentation of the sheet and its transient shedding is strongly non-uniform in circumferential direction. The collapse of the vapor structures is enforced by the positive pressure gradient in radial direction.

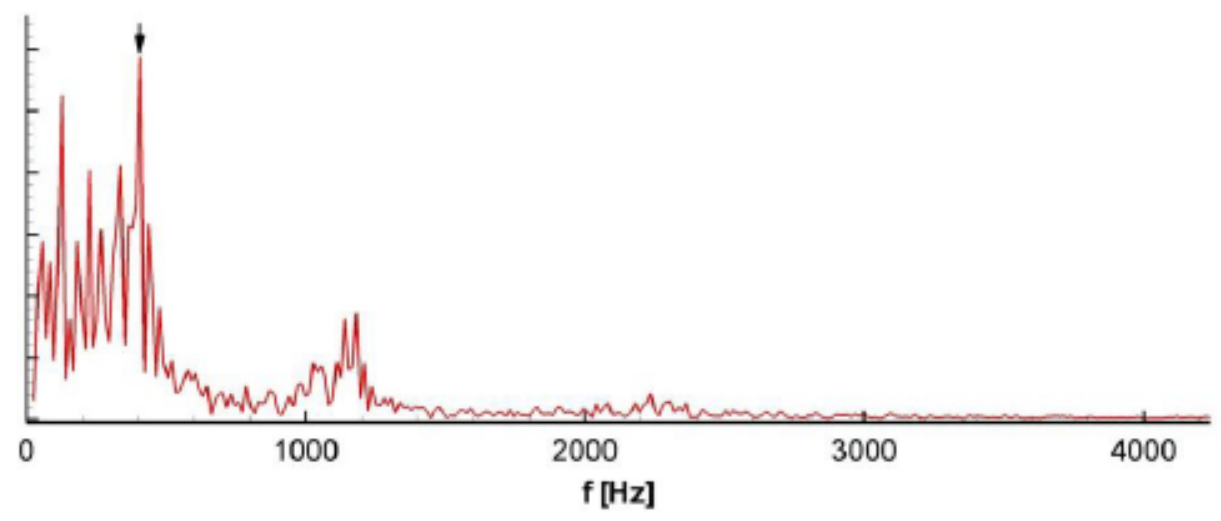

Figure 5: FFT of the time history of the vapor volume fraction. 

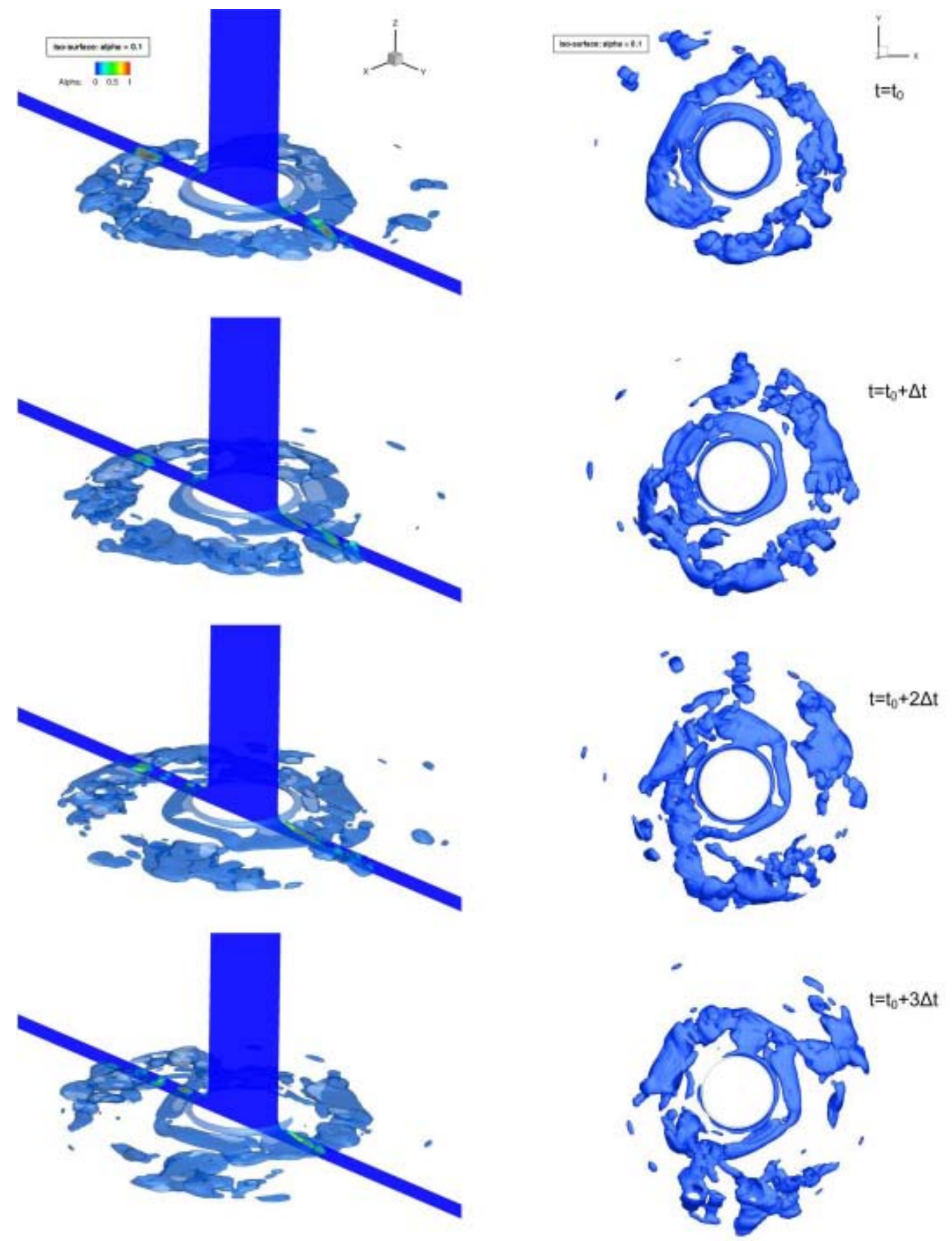

Figure 6: Iso-surfaces of the vapor volume fraction $(\alpha=0.1)$ at 4 equidistant instants in time $\left(\Delta \mathrm{t}=8.4 \cdot 10^{-5} \mathrm{~s}\right)$. Left side: Perspective view with an additional axial cut plane showing the contour of the vapor volume fraction. Right side: Top view. 
Figure 5 depicts the spectrum of the integrated vapor volume. The dominant frequency is $408 \mathrm{~Hz}$ (arrow), which corresponds to the radial shedding. Lower frequencies might be circumferential modes. The highest well-defined frequencies at $1139 \mathrm{~Hz}$ and $1182 \mathrm{~Hz}$ are supposed to be related to the collapse of small clouds.
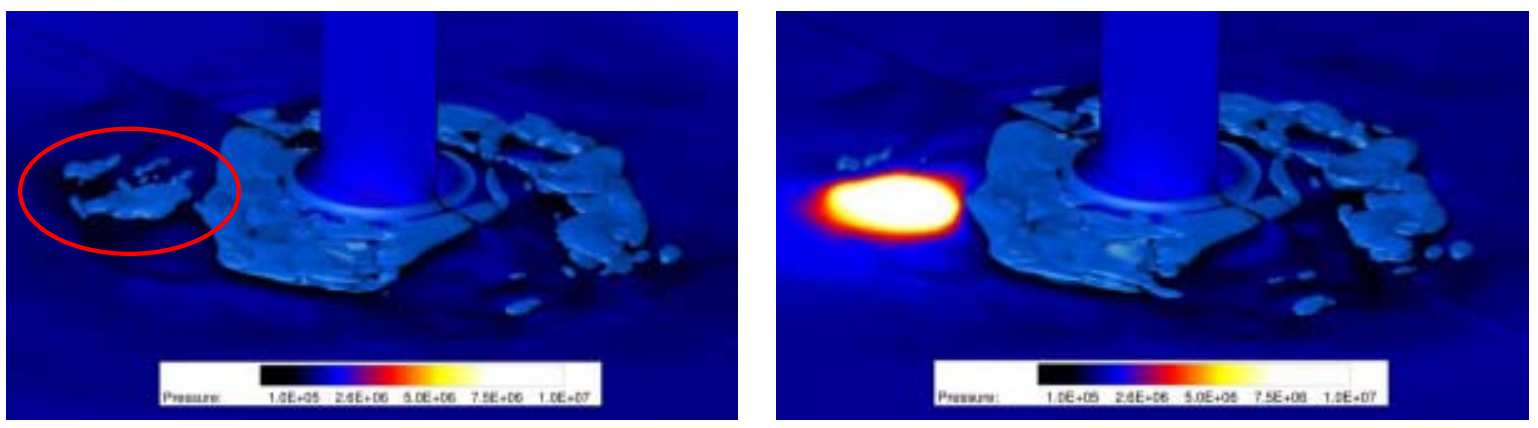

Figure 7: Perspective view of two time instants $(\Delta t=28 \mu \mathrm{s})$ showing the collapse of a vapor structure and the resulting shock. Vapor structures are indicated by iso-surfaces $(\alpha=0.1)$. The color on the target wall and on the axial cut plane shows the static pressure.

Figure 7 shows a perspective view of two consecutive instants in time $\left(\Delta \mathrm{t}=2.8 \cdot 10^{-5} \mathrm{~s}\right)$. On the left side, a couple of vapor structures marked in red are observed at a radial position of $\approx 2.6 \cdot 10^{-2} \mathrm{~m}$. On the right side, most of these vapor structures have already collapsed and a shockwave has formed. Hence, the duration of the complete collapse is about $2.8 \cdot 10^{-5} \mathrm{~s}$, which is in our case resolved by approximately 1000 time steps.

Figure 8a shows a photograph (from an angle) of an eroded target disc. The damage exhibits an almost perfect circular shape. The red circles indicate the area where damage was found in the experiment. ${ }^{8}$
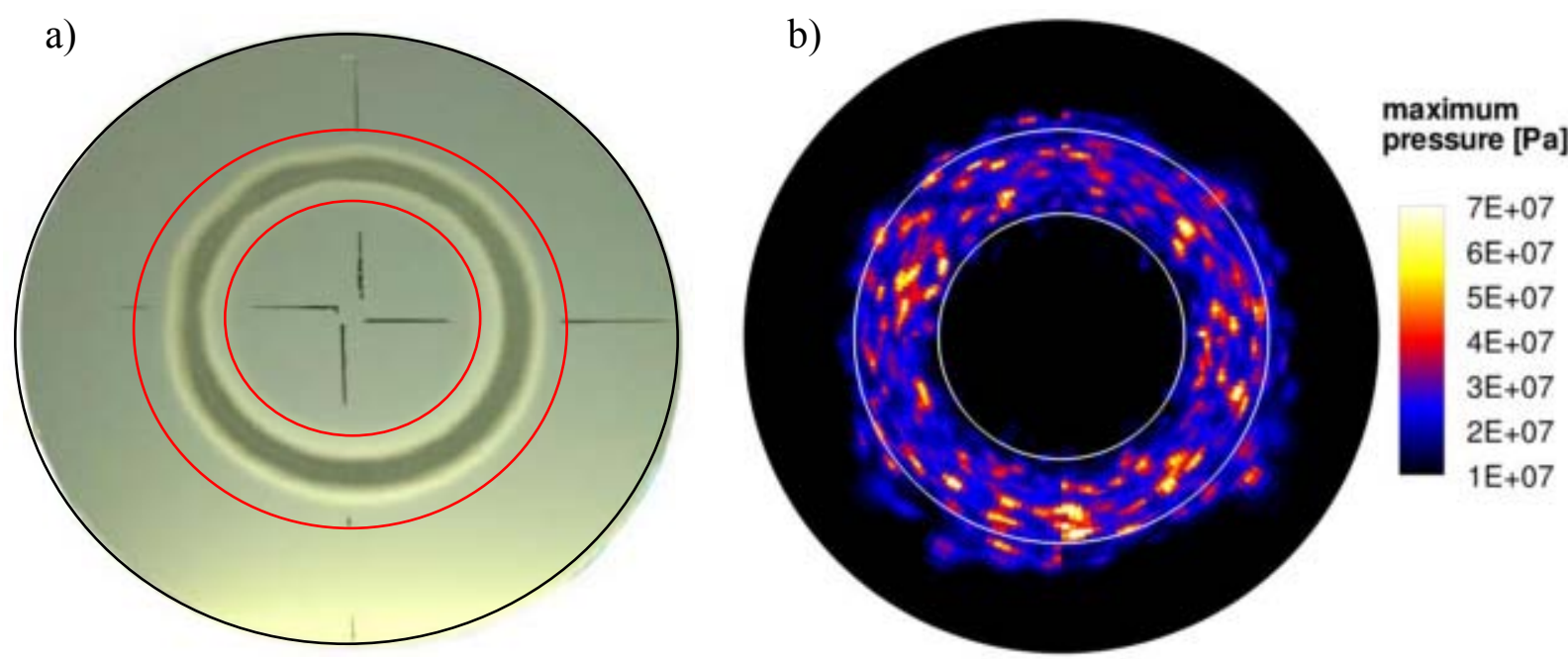

Figure 8: a) Photograph of an eroded target from the experiment - slightly perspective view. ${ }^{8}$ According to experimental observation, erosion damage occurs within $\mathrm{r}=19 \mathrm{~mm}$ and $\mathrm{r}=32 \mathrm{~mm}$ (red circles). ${ }^{8} \mathrm{~b}$ ) Maximum pressure observed in the simulation on the target during the analysis interval. 
Figure $8 \mathrm{~b}$ shows the maximum pressure for each computational cell on the target disc recorded during the analysis interval (interval 3). Only pressures about one order of magnitude higher than the stagnation pressure are shown, the highest recorded value is $1.56 \cdot 10^{8} \mathrm{~Pa}$. We observe a convincing analogy of the cell-wise maximum pressure and the experimentally detected areas of erosion damage. However, further investigations using much larger analysis intervals are required to improve the statistical relevance of the prediction.

In the following subsection we present the results of the "collapse detector". Since the detected minimum divergence is comparable to the detected maximum pressure for the investigated test cases, we focus on the presentation of the maximum collapse pressure exclusively. However these two quantities may not necessarily be equivalent in other test cases.

Figure 9 shows the collapses detected within the gap between the nozzle and the target disc during the analyzed part of the simulation. The collapses are indicated as spheres that are plotted at the position of their occurrence. The diameter of each sphere and its color represent the collapse intensity. On the left side (a), the collapse pressure $p_{\text {collapse }}$ is visualized and on the right side (b), the maximum pressure referred to the target wall $p_{\text {wall }}$ (Eq. 1) is depicted. In both cases most of the collapses are found within the marked area.
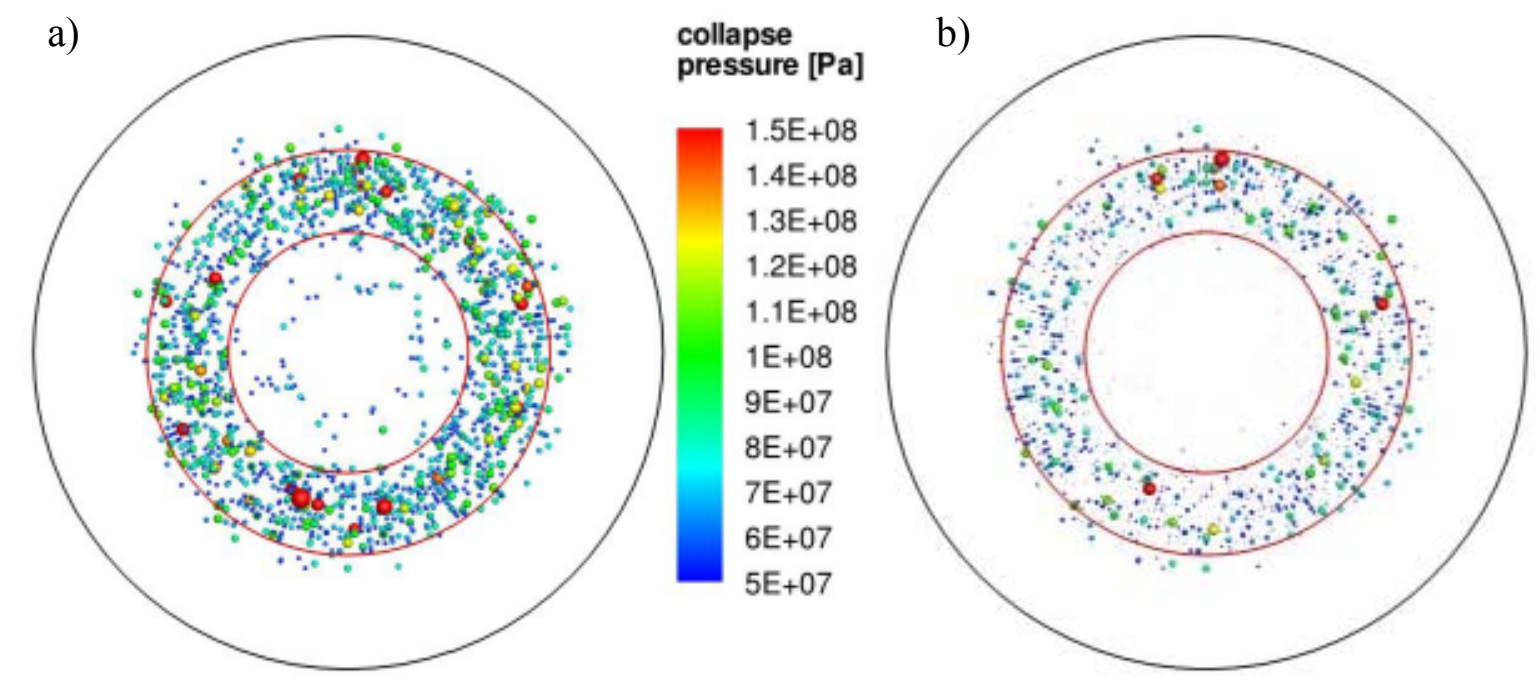

Figure 9: Visualization of all collapses detected during the analysis interval $\left(\Delta \mathrm{t}=7 \cdot 10^{-2} \mathrm{~s}\right)$. Each collapse is represented by a sphere at the position of its occurrence. Size and color represent the collapse intensity. On the left side, the maximum pressure at the collapse center is shown - on the right side, the collapse pressure referred to the surface of the target is depicted.

Compared to Figure 9a, in Figure 9b the agreement with the experiment even improves when the collapse pressure is projected to the target. 


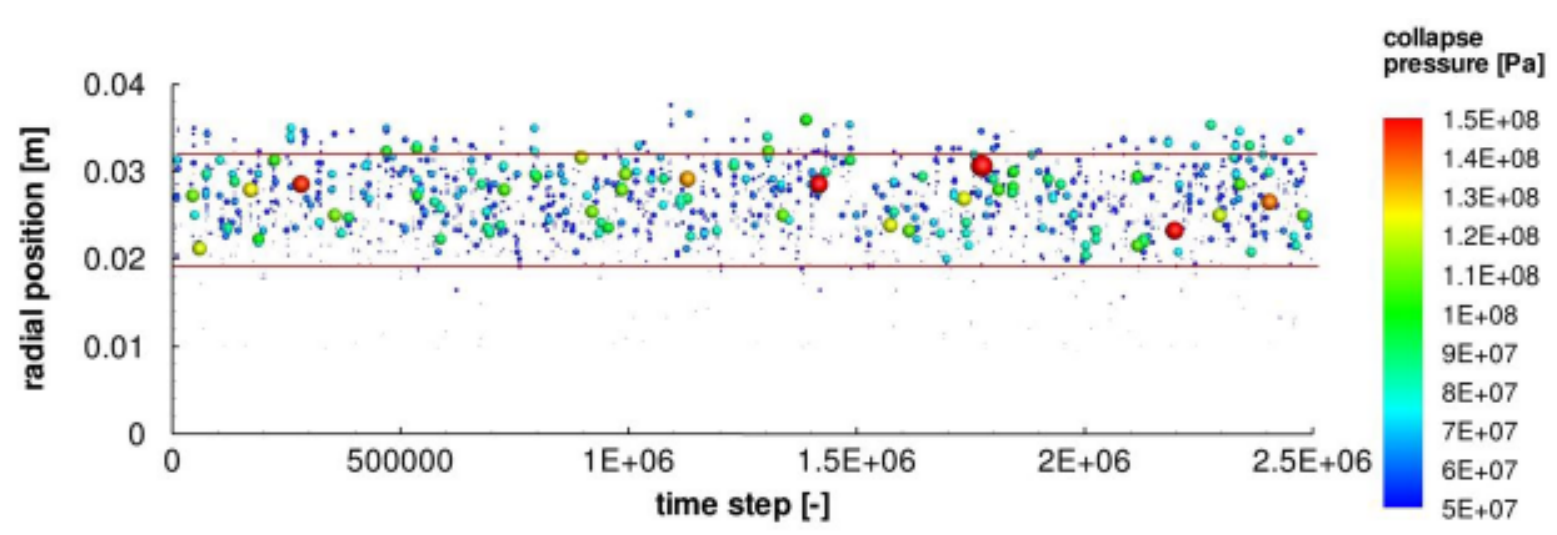

Figure 10: Time history of collapses detected during the analysis interval. The diagram shows the radial position of each detected collapse against the time of its occurrence. Size and color of the collapses represent the collapse pressure referred to the surface of the target.

Figure 10 shows the radial positions of all collapses plotted against the time of their occurrence. According to Figure 9b, size and color of each sphere represent the collapse pressure referred to the target wall $\left(p_{\text {wall }}\right)$. One observes that all violent collapses (red and yellow spheres) are located within the marked area. The lower bound at $r=19 \mathrm{~mm}$ is perfectly captured by the simulation while the upper bound might be slightly overestimated. Assuming that the positions of collapses are statistically distributed in circumferential direction, this time history represents a possible load profile the material might be exposed to over a long time period.

Table 1 quantifies the observed collapses with respect to their intensity and frequentness. One observes that the number of collapse events strongly decreases when the pressure threshold is increased. As expected, the number of collapses decreases significantly as well, when the collapse pressure is referred to the surface of the target (Table 2). This data may offer a possibility for calibrating thresholds for the collapse pressure by making a comparison of the numerically predicted loads to experimental pitting tests and pit counts.

\begin{tabular}{|c|c|c|}
\hline collapse pressure $>$ & number of collapse events & events per time unit \\
\hline $1.5 \times 10^{8} \mathrm{~Pa}$ & 11 & $1571 / \mathrm{s}$ \\
\hline $1.25 \times 10^{8} \mathrm{~Pa}$ & 31 & $4421 / \mathrm{s}$ \\
\hline $1.0 \times 10^{8} \mathrm{~Pa}$ & 139 & $19861 / \mathrm{s}$ \\
\hline $0.75 \times 10^{8} \mathrm{~Pa}$ & 498 & $71141 / \mathrm{s}$ \\
\hline
\end{tabular}

Table 1: Collapse events in the whole domain per time unit

\begin{tabular}{|c|c|c|}
\hline $\begin{array}{c}\text { collapse pressure referred to } \\
\text { the surface of the target }>\end{array}$ & number of collapse events & events per time unit \\
\hline $1.5 \times 10^{8} \mathrm{~Pa}$ & 2 & $291 / \mathrm{s}$ \\
\hline $1.25 \times 10^{8} \mathrm{~Pa}$ & 4 & $581 / \mathrm{s}$ \\
\hline $1.0 \times 10^{8} \mathrm{~Pa}$ & 12 & $1701 / \mathrm{s}$ \\
\hline $0.75 \times 10^{8} \mathrm{~Pa}$ & 65 & $9281 / \mathrm{s}$ \\
\hline
\end{tabular}

Table 2: Collapse events in the whole domain per time unit. The collapse pressure is referred to the surface of the target. 


\section{CONCLUSIONS}

We present a novel approach referred to as "collapse detector" for the numerical prediction of cavitation erosion. Based on a set of physical criteria we detect and characterize isolated collapses of vapor structures within unsteady cavitating flows. The collapse detector is implemented into our flow solver CATUM, which enables the simulation of cloud collapses and the subsequent formation and propagation of shocks due to the compressible treatment of the working fluid. Through a comparison of our results with experimental data we demonstrate the ability of the proposed methodology to predict the position of cavitation erosion. Additionally we provide information about the relative strength and the time history of collapse events. Currently, the significance of solved gas and its effect on cavitation erosion are beeing investigated. Further research will focus on the comparison of our numerical predictions to experimental pitting tests, as well as the application of our collapse data to a material law as proposed by Karimi ${ }^{8}$.

\section{ACKNOWLEDGEMENTS}

We would like to thank Jean-Pierre Franc for providing detailed information on his experimental setup as well as for fruitful discussions and suggestions. This work was partly funded by the DFG (Deutsche Forschungsgemeinschaft).

\section{REFERENCES}

[1] C.E. Brennen, Cavitation and Bubble Dynamics, Oxford University Press, New York, 1995

[2] J.-P Franc and J.-M. Michel, Fundamentals of Cavitation, Kluwer Academic, Dordrecht, 2004

[3] G.H. Schnerr, I.H. Sezal and S.J. Schmidt, Numerical Investigation of Three-dimensional Cloud Cavitation with Special Emphasis on Collapse Induced Shock Dynamics, Physics of Fluids, Vol. 20, Issue 4, 040703, 2008.

[4] S.J. Schmidt, M. Thalhamer amd G.H. Schnerr, Inertia Controlled Instability and Small Scale Structures of Sheet and Cloud Cavitation, Proc. 7th CAV 2009 - 7th International Symposium on Cavitation, Ann Arbor, Michigan, USA, 16.8.- 21.8.2009, paper 17, CDROM publication, 2009.

[5] S.J. Schmidt, I.H. Sezal, G.H. Schnerr and M. Thalhamer, Numerical Analysis of Shock Dynamics for Detection of Erosion Sensitive Areas in Complex 3-D Flows, Proc. WIMRC Cavitation Forum 2008, pp. 107-120, July 7 - 9, 2008, Warwick, UK, 2008.

[6] S.J. Schmidt, M. Mihatsch, M. Thalhamer, C. Egerer, S. Hickel and N.A. Adams, Assesment of Erosion Sensitive Areas via Compressible Simulation of Unsteady Cavitating Flows, International Workshop on ADVANCED EXPERIMENTAL AND NUMERICAL TECHNIQUES FOR CAVITATION EROSION PREDICTION, Grenoble, France, 1.3- 2.3.2011, Post-Workshop CD.

[7] S.J. Schmidt, M. Mihatsch, M. Thalhamer, N.A. Adams, Assessment of the Prediction Capability of a Thermodynamic Cavitation Model for the Collapse Characteristics of a Vapor-Bubble Cloud, WIMRC Cavitation Forum 2011, Warwick, UK. 
[8] J.P. Franc, M. Riondet, Incubation Time and Cavitation Erosion Rate of Work-Hardening Materials, CAV2006, Wageningen, The Netherlands, September 2006.

[9] A. Karimi, W.R. Leo, Phenomenological Model for Cavitation Erosion Rate Computation, Materials Science and Engineering, 95 (1987) 1-14 